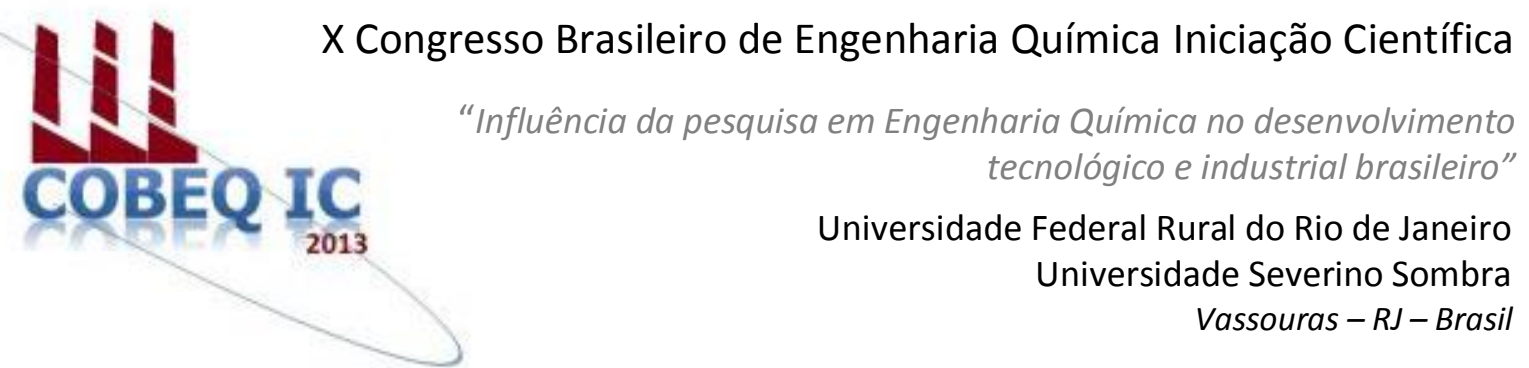

\title{
DETERMINAÇÃO DOS PARÂMETROS DE INTERAÇÃO DE ALCOÓIS EM PDMS E PA POR SPME/GC
}

\author{
ROSA $^{* 1}$, G.S.; ELIAS ${ }^{2}$, A.M.; COELHO ${ }^{3}$, G.L.V. \\ ${ }^{1}$ Aluno do DEQ/UFRRJ $\quad{ }^{2}$ Mestrando do DEQ/UFRRJ $\quad{ }^{3}$ Professor do DEQ/UFRRJ \\ Departamento de Engenharia Química - Universidade Federal Rural do Rio de Janeiro \\ Endereço-UFRRJ, BR 465, km 07, Seropédica, CEP. 23.890-000, RJ, \\ email: coelho@ufrrj.br
}

\begin{abstract}
RESUMO - Neste estudo a micro extração em fase sólida (SPME, solid phase micro extraction) foi utilizada para a obtenção dos parâmetros de Flory Huggins em sistemas de etanol e metanol nas fibras de Polidimetilsiloxano (PDMS) e Poliacrilato (PA). Verificou-se a veracidade de algumas teorias que apontam uma relação entre $\mathrm{o}$ parâmetro e a temperatura. Para isso, considerou-se cinco distintas temperaturas, de $323,15 \mathrm{~K}$ a 363,15K. A relação foi comprovada.
\end{abstract}

Palavras chave: Termodinâmica, Solubilidade, Cromatografia

\section{INTRODUÇÃO}

Atualmente os polímeros estão presentes por toda parte, em praticamente tudo que utilizamos, como pratos, talheres, automóveis, descartáveis, etc. Consequentemente, o investimento em desenvolvimento de novos materiais é constante. Neste sentido, um parâmetro importante para a indústria química, principalmente a polimérica, é o parâmetro de Flory-Huggins, que é um parâmetro de solubilidade específico para um determinado polímero em um determinado solvente. Seu valor é inversamente proporcional à solubilidade de um polímero em um solvente, ou seja, quanto maior o valor do parâmetro de Flory-Huggins, menor é a solubilidade do polímero. Algumas teorias afirmam que esse parâmetro é dependente da temperatura e também é função da composição do sistema e da massa molecular do polímero.
Este trabalho tem como objetivo determinar valores inéditos do parâmetro de Flory Huggins para alcoóis em PDMS e Poliacrilato em cinco temperaturas prédeterminadas $(323,15 \mathrm{~K} ; 333,15 \mathrm{~K} ; 343,15 \mathrm{~K}$; $353,15 \mathrm{~K} ; 363,15 \mathrm{~K})$. Na primeira parte do projeto, já foi possível a determinação do parâmetro para o etanol em PDMS e Poliacrilato. Na segunda visou-se, então, estabelecer o parâmetro também para o metanol em PDMS e butanol em Poliacrilato. Para isso, novamente foi utilizada a técnica de SPME (microextração por fase sólida) acoplada à cromatografia gasosa. Desta maneira, foi possível verificar a existência da real dependência do parâmetro com a temperatura, como afirmam algumas teorias.

\section{Cromatografia gasosa}

A cromatografia gasosa é uma técnica reconhecida e uma ferramenta poderosa de 
quantificação analítica. Sob condições próprias e em temperaturas relativamente altas, esta técnica resulta em uma boa fonte de dados termodinâmicos de soluções em diluição infinita, principalmente no campo polimérico. Segundo a literatura, é possível determinar com essa técnica analítica volumes de retenção e entalpia padrão parcial molar de vaporização com incertezas de $0,3 \%$ e $1 \%$, respectivamente. Por ser uma técnica de grande precisão analítica, a cromatografia gasosa é uma forte aliada à técnica de SPME (Hammer e Ligny, 1974).

Os experimentos cromatográficos fornecem um parâmetro de retenção que requer um modelo para expressar os resultados em uma forma adequada para interpretação das propriedades do material, como na determinação do parâmetro de Flory-Huggins, onde a quantificação do volume de retenção é fundamental para o cálculo do parâmetro.

\section{SPME - Microextração por fase sólida}

A SPME é uma microtécnica em que os processos de extração e pré-concentração de analitos ocorrem e escala dimensional mínima, que não é das mais usuais. Além disso, não utiliza solvente e tem alto poder de concentração. Como mostrado na figura 1, o dispositivo básico de SPME consiste de um bastão de fibra ótica, de sílica fundida de $100 \mu \mathrm{m}$ de diâmetro, com $10 \mathrm{~mm}$ de uma extremidade recoberta com um fino filme de um polímero adsorvente (a fase estacionária) de diferentes espessuras e tipos, como por exemplo, o polidimetilsiloxano (PDMS) e o poliacrilato (PA). A extremidade da sílica pode ser recoberta também com um sólido adsorvente, como o carvão ativo.

As seguintes considerações ilustram as vantagens da microextração. Imagine uma gotícula, de $1 \mu \mathrm{L}$ de solvente orgânico colocada em $10 \mathrm{~mL}(10.000 \mu \mathrm{L})$ de uma matriz aquosa com analitos orgânicos. Esta gotícula de solvente representaria um décimo de milésimo do volume da matriz. Por afinidade, analitos orgânicos migrariam da matriz para a gotícula de solvente onde seriam concentrados de acordo com seus coeficientes de partição entre o solvente orgânico e a matriz aquosa. Para fins quantitativos, depois de saturada com os analitos, a gotícula de $1 \mu \mathrm{L}$ teria de ser retirada intacta do seio da matriz - onde ela deveria ser imiscível, mesmo sob agitação - e injetada por Cromatografia Gasosa. O volume da gota e o nível de concentração dos analitos resultariam em um cromatograma em que a superposição dos analitos à cauda do pico do solvente não comprometeria a análise quantitativa.

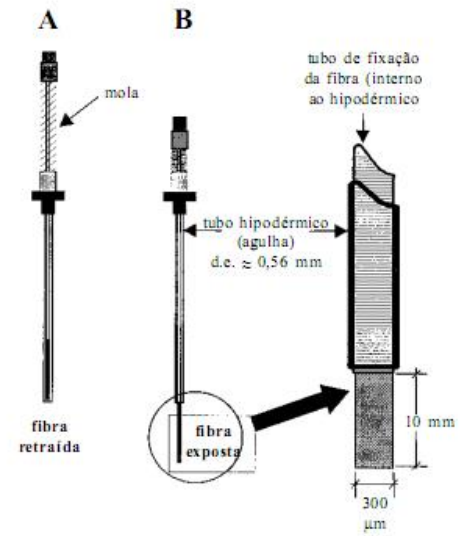

\section{Figura 1 - Dispositivo da fibra de SPME}

A microescala apresentaria mais uma vantagem. Como, em comparação à matriz, em cada gota haveria pouco analito, as pequenas quantidades extraídas não descaracterizariam as correspondentes concentrações na matriz. Desta forma, outras extrações poderiam ser realizadas na mesma matriz, para dispor de replicatas estatisticamente confiáveis. Entretanto, na forma descrita acima, este procedimento seria inviável pelas dificuldades experimentais inerentes, por exemplo, à manipulação de volumes tão pequenos de solvente (Valente e Augusto, 2000).

Na SPME, o recobrimento da fibra age de modo similar à gotícula do exemplo acima. Entretanto, por estar imobilizado no suporte de sílica fundida, a sua introdução e remoção quantitativa da matriz é facilitada, contornando grande parte dos problemas práticos associados ao exemplo discutido. Porém, sob os demais aspectos a SPME mantém todas as demais complexidades inerentes aos métodos de extração convencionais.

A aplicação dos fundamentos de transferência de massa e da termodinâmica é o pilar fundamental da SPME. O método é baseado em equilíbrios multifásicos, no qual são consideradas usualmente três fases: a fibra extratora, a fase gasosa, conhecida como headspace, e a matriz homogênea, entre as 
quais, durante a extração, os analitos migram até que o equilíbrio seja alcançado, onde se tem a igualdade dos potencias químicos $\left(\mu_{\mathrm{f}}=\right.$ $\mu_{\mathrm{g}}=\mu_{\mathrm{s}}$ ) entre as fases, respectivamente (Fonseca e Coelho, 2007).

A microextração por fase sólida comumente é realizada no modo headspace. Este procedimento consiste em colocar uma amostra no frasco, esperar as fases líquida e/ou sólida entrarem em equilíbrio com a fase gasosa e, então, expor a fibra somente na região do topo do frasco, extraindo os analitos gasosos. Esta técnica tem sido utilizada, por exemplo, para determinar pesticidas organoclorados em diferentes matrizes, como água, solo e fluidos biológicos, pois confere maior durabilidade à fibra por não entrar em contato com a amostra.

\section{O parâmetro de Flory e Huggins}

A termodinâmica clássica de sistemas binários polímero-solvente foi desenvolvida independentemente por P. J. Flory e M. L. Huggins. A teoria de Flory-Huggins captura as características essenciais de uma mistura e a magnitude das mudanças entrópicas e entálpicas da mesma, fornecendo uma base de conhecimento há mais de cinquenta anos.

A teoria de Flory-Huggins é baseada no conhecimento do modelo quantitativo de estrutura formulado por K. H. Meyer. Meyer apontou que a diferença molecular entre polímero e solvente produzia consequências na entropia de mistura. O cálculo quantitativo da entropia de mistura levou à introdução de um parâmetro adimensional, chamado de parâmetro de interação de Flory-Huggins $(\chi)$, usado para descrever a termodinâmica das soluções poliméricas (Brandrup et al., 1998).

O parâmetro de Flory-Huggins é um importante fator de miscibilidade entre polímeros e solventes. É utilizado para analisar a compatibilidade entre estes, sendo que quanto menor é o seu valor, maior é a compatibilidade entre polímeros e solventes. Um valor de $\chi<0,5$ é um critério da teoria de Flory-Huggins para um polímero e solvente serem completamente miscíveis na faixa de composição global. Este parâmetro aparece na mais importante equação da Teoria de FloryHuggins que relaciona pressão de vaporização da solução polimérica com pressão de vapor do solvente puro (Ovejero et al., 2007).

Este parâmetro de interação pode ser determinado por diversos métodos, como espalhamento de nêutrons a baixos ângulos, calorimetria diferencial de varredura, cromatografia gasosa inversa, cromatografia gasosa, que são relatados em diferentes artigos. Dentre estas técnicas utilizadas, a mais consolidada é a Cromatografia Gasosa Inversa (Voelkel et al., 2009).

Nos estudos de Paik e Writer (1995), os resultados mostraram que o modelo de FloryHuggins descreve bem a sorção de flavors em materiais poliméricos. Considerou-se a contribuição entrópica devido à diferença de tamanhos entre as moléculas poliméricas e a dos flavors. Contudo, apesar de apresentar uma melhor acurácia dos resultados, o modelo de Flory-Huggins apresentou grandes erros em relação aos dados experimentais, sendo usado apenas como um modelo qualitativo na descrição da sorção e flavors em materiais poliméricos.

Kalyuzhnyi e Yampol'skill (1989), estudando sistemas de poliviniltrimetilsilano (PVTMS)-hidrocarbonetos e de PVTMSálcoois, notaram que a dependência do parâmetro de Flory-Huggins $(\chi)$ com a temperatura era diferente nos dois sistemas. Enquanto que no sistema de PVTMShidrocarbonetos o parâmetro aumentava com a temperatura, tornando os hidrocarbonetos solventes ruins ( $\theta$ solventes), no sistema de PVTMS-álcoois o valor do parâmetro diminuía, favorecendo a interação polímerosolvente. Segundo os autores, essa tendência pode ser devida ao fato de haver uma baixa temperatura critica de dissolução para o sistema PVTMS-hidrocarbonetos, no qual em temperaturas maiores a partir dessa temperatura critica, a interação polímerosolvente é desfavorecida, enquanto que no sistema PVTMS-álcoois pode haver uma alta temperatura critica de dissolução, onde a interação polímero solvente é favorecida em temperaturas maiores que a temperatura critica de dissolução. 


\section{FUNDAMENTOS TEÓRICOS}

Numa extração por SPME, as moléculas do analito têm de se deslocar da matriz e penetrar no recobrimento. Para isto, resistências a transferências de massa devem ser vencidas até que se estabeleça um equilíbrio de partição (ou de adsorção para o caso de recobrimentos sólidos) do analito entre a fibra e o meio que a envolve.

Segundo Valente e Augusto (2000), o coeficiente de partição fibra-matriz $\left(\mathrm{K}_{\mathrm{fh}}\right)$ é dado pela Equação 1, onde, $\mathrm{C}_{\mathrm{f}}$ é a concentração do analito na fibra e $C_{m}$ é a concentração do analito na matriz.

$\mathrm{K}_{\mathrm{fh}}=\mathrm{C}_{\mathrm{f}} / \mathrm{C}_{\mathrm{m}}$

A Equação 2 mostra a relação entre o coeficiente de partição de um determinado soluto e o volume de retenção do mesmo (Martos et al., 1997).

$\mathrm{V}_{\mathrm{g}}=\mathrm{K} / \rho$

Onde $\mathrm{V}_{\mathrm{g}}$ é o volume de retenção do analito, $\mathrm{K}$ é o coeficiente de partição e $\rho$ é a densidade do polímero.

$\mathrm{O}$ termo $\mathrm{V}_{\mathrm{g}}$ desempenha um papel importante no cálculo dos parâmetros termodinâmicos e das energias de interação. A energia de interação entre um polímero e um soluto é expressa em termo de energia livre de mistura $\Delta \mathrm{G}_{\text {mix }}$, o qual é o termo chave da miscibilidade de um sistema polímero-soluto.

Um valor negativo de $\Delta \mathrm{G}_{\text {mix }}$ indica que o sistema polímero-soluto é miscível. Se $\varphi_{\mathrm{i}}$ é a fração volumétrica do i-ésimo componente, então a energia livre de mistura pode ser descrita pela teoria de Flory-Huggins segundo a Equação 3 (Heberguer et al., 2005):

$\Delta \mathrm{G}_{\text {mix }}=\mathrm{RT}\left(\mathrm{n}_{1} \ln \varphi_{1}+\mathrm{n}_{2} \ln \varphi_{2}+\mathrm{n}_{1} \varphi_{2} \chi\right)$

A relação entre o coeficiente de atividade em diluição infinita do solvente $\left(\Omega_{1}{ }^{\infty}\right)$ e o volume de retenção $\left(V_{\mathrm{g}}, \mathrm{cm}^{3} / \mathrm{g}\right)$, é dada pela Equação 4, onde $\mathrm{T}$ é a temperatura em $\mathrm{K}, \mathrm{R}$ é a constante universal dos gases, $\mathrm{M}_{1}$ é o peso molecular do solvente, $\mathrm{P}_{1}{ }^{0}$ é a pressão de saturação do solvente, $V_{1}$ é o volume molar do solvente $\left(\mathrm{cm}^{3} / \mathrm{mol}\right)$ e $B_{11}$ é o segundo coeficiente do virial $\left(\mathrm{cm}^{3} / \mathrm{mol}\right)$ (Conder e Young, 1979).

$\ln \left(\Omega_{1}{ }^{\infty}\right)=\ln \left[\mathrm{R} \cdot \mathrm{T} /\left(\mathrm{V}_{\mathrm{g}} \cdot \mathrm{M}_{1} \cdot \mathrm{P}_{1}{ }^{0}\right)\right] \quad-\quad\left(\mathrm{B}_{11^{-}}\right.$ $\left.\mathrm{V}_{1}\right) \cdot \mathrm{P}_{1}^{0} /(\mathrm{R} \cdot \mathrm{T})$

(4)

Dos valores dos coeficientes de atividade em diluição infinita, o parâmetro de Flory e Huggins pode ser calculado através da Equação 5 abaixo, onde $r$ é a razão entre o volume molar do polímero e o volume molar do solvente, e $\rho_{1} \mathrm{e} \rho_{2}$ são as densidades do solvente e do polímero, respectivamente (Flory, 1953).

$$
\chi=\ln \left(\Omega_{1}{ }^{\infty}\right)-(1-1 / \mathrm{r})+\ln \left(\rho_{1} / \rho_{2}\right)
$$

\section{MATERIAIS E MÉTODOS}

A obtenção dos parâmetros consistiu em duas etapas fundamentais: extração dos alcoóis por SPME e confecção da curva padrão. Todos os reagentes utilizados foram obtidos da Vetec Química Fina Ltda., sendo estes: etanol, metanol e o-xileno, todos com pureza superior a 99.3\%. Para a técnica de SPME, foram utilizadas fibras de poliacrilato e de PDMS com espessuras de $85 \mu \mathrm{m}$ e $30 \mu \mathrm{m}$, respectivamente, ambas obtidas da Supelco. Frascos âmbar de $44 \mathrm{~mL}$ com septo de PTFE/silicone foram utilizados para confinamento e estudo do analito. $\mathrm{O}$ cromatógrafo a gás utilizado para análise foi um GC-2010 Shimadzu equipado com detector de ionização em chama, uma coluna capilar HP-Innowax (polietileno glicol reticulado; crosslinked PEG) de dimensões $60 \mathrm{~m}$ x 0,32 $\mathrm{mm} \times 0,25 \mu \mathrm{m}$; o liner utilizado foi obtido de SGE Analytical Science Pty Ltd (próprio para SPME), sendo utilizado como gás de arraste o hélio ultrapuro $(99,999 \%)$. As fibras de SPME utilizadas foram condicionadas no injetor do cromatógrafo a gás a temperatura de $250{ }^{\circ} \mathrm{C}$ por 40 minutos.

O tempo de extração foi determinado preparando amostras gasosas através da injeção de $1 \mu \mathrm{L}$ a $3 \mu \mathrm{L}$ de analito puro em frascos âmbar de $44 \mathrm{~mL}$ vedados com septo PTFE/silicone. A temperatura no interior dos frascos foi mantida constante por um banho termostático (Lauda, modelo RM 6B) com precisão de $0,1 \mathrm{~K}$. Depois de injetado o analito 
e a temperatura ter sido mantida constante, a fibra foi exposta no interior do recipiente por tempos determinados e, depois de transcorrido o tempo de exposição, levada ao injetor do cromatógrafo para quantificação do material extraído. Os experimentos foram realizados aumentando-se o tempo de exposição até que a massa extraída não variasse mais com o aumento do período de exposição, indicando que o equilíbrio fora alcançado.

Para a determinação dos coeficientes de partição entre a fase polimérica da fibra de SPME e o solutos na fase gasosa para os três sistemas estudados, nas cinco temperaturas, foram injetados $3 \mu \mathrm{L}$ do analito desejado (metanol ou etanol) nos frascos âmbar e o sistema foi mantido a temperatura constante em um banho termostático . A fibra foi exposta à amostra durante o tempo de extração previamente determinado e, então, levado ao injetor do cromatógrafo a gás para quantificação do material extraído. Para a obtenção da curva de calibração, preparou-se diferentes soluções com concentrações conhecidas de analitos em o-xileno e injetouse diretamente no cromatógrafo diferentes quantidades com uma seringa para que se pudesse fazer a correlação entre as áreas obtidas pela SPME e as massas as quais correspondiam. Todas as análises foram realizadas em iguais condições cromatográficas: pressão no injetor constante a $113 \mathrm{kPa}$, fluxo total de $12 \mathrm{~mL} / \mathrm{min}$, fluxo na coluna de $2 \mathrm{~mL} / \mathrm{min}$, velocidade linear de 27,8 $\mathrm{cm} / \mathrm{s}$, purga zero e razão de split igual a 5 .

\section{RESULTADOS E DISCUSSÕES}

Os tempos de extração do etanol pelas fibras de PDMS e Poliacrilato foram iguais a 3 minutos e a 12 minutos, respectivamente. Já o tempo de extração do metanol-PDMS foi de 5 minutos e do butanol-PA, 20 minutos. Essa diferença entre os tempos de extração da fibra de PDMS e Poliacrilato se deve ao fato das fibras apresentarem diferentes volumes e diferentes polaridades.

A tabela 1 mostra os resultados obtidos para o sistema de Polidimetilsiloxano em etanol. Observa-se que os valores dos parâmetros de Flory-Huggins apresentam-se inversamente proporcionais à temperatura.
Com isso, a solubilidade está aumentando com o incremento de temperatura.

Tabela 1 - Parâmetros termodinâmicos para o sistema PDMS-Etanol

\begin{tabular}{clllll}
\hline $\mathbf{T}(\mathbf{K})$ & 323,15 & 333,15 & 343,15 & 353,15 & 363,15 \\
\hline $\mathbf{K}_{\mathbf{f m}}$ & 40,00 & 31.60 & 25.40 & 21,50 & 17,00 \\
$\mathbf{V}_{\mathbf{g}}$ & 40,90 & 32,30 & 26,00 & 22,00 & 17,40 \\
$\mathbf{\Omega}_{\mathbf{1}}^{\infty}$ & 40,30 & 40,60 & 34,00 & 27,80 & 25,00 \\
$\mathbf{X}$ & 2,63 & 2,42 & 2,23 & 2,01 & 1,89 \\
\hline
\end{tabular}

A tabela 2 apresenta o estudo do sistema PA-etanol. O valor do parâmetro de FloryHuggins diminui com a temperatura, ou seja, a miscibilidade aumenta. Este fato também ocorreu no sistema etanolpoliviniltrimetilsilano estudado por KalyuzhnyieYampol'skill (1989) e, além disso, como era de se esperar, a miscibilidade da fibra de poliacrilato em etanol é maior que a da fibra de PDMS, visto que há uma maior semelhança de polaridade entre o álcool e a fibra de poliacrilato.

Tabela 2 - Parâmetros termodinâmicos para o sistema Poliacrilato-Etanol

\begin{tabular}{clllll}
\hline $\mathbf{T}(\mathbf{K})$ & 323,15 & 333,15 & 343,15 & 353,15 & 363,15 \\
\hline $\mathbf{K}_{\mathbf{f m}}$ & 95,10 & 64,90 & 45,60 & 33,30 & 24,10 \\
$\mathbf{V}_{\mathbf{g}}$ & 95,10 & 64,90 & 45,60 & 33,30 & 24,10 \\
$\mathbf{\Omega}_{\mathbf{1}}$ & 21,20 & 20,10 & 19,20 & 18,20 & 17,90 \\
$\mathbf{X}$ & 1,77 & 1,70 & 1,64 & 1,57 & 1,53 \\
\hline
\end{tabular}

A tabela 3 apresenta os dados obtidos para o metanol em PDMS. Diante dos altos valores do parâmetro encontrado, constatou-se uma baixa miscibilidade entre o polímero e o solvente. Isto ocorre devido à diferença de polaridade entre os componentes. Ao contrário dos sistemas anteriores, nesse a relação entre $\chi$ e a temperatura é diretamente proporcional, ou seja, com o aumento de temperatura, o parâmetro aumenta e, consequentemente, a solubilidade diminui.

Tabela 3 - Parâmetros termodinâmicos para o sistema PDMS-Metanol

\begin{tabular}{cccccc}
\hline $\mathbf{T ~ K} \mathbf{~}$ & 323,15 & 333,15 & 343,15 & 353,15 & 363,15 \\
\hline $\mathbf{K}_{\mathbf{f m}}$ & 1,50 & 1,24 & 0,96 & 0,71 & 0,67 \\
$\mathbf{V}_{\mathbf{g}}$ & 1,53 & 1,27 & 0,98 & 0,73 & 0,68 \\
$\mathbf{\Omega}_{\mathbf{1}}^{\infty}$ & 2,83 & 3,53 & 4,68 & 6,51 & 7,11 \\
$\mathbf{X}$ & 41,74 & 41,96 & 42,25 & 42,58 & 42,67 \\
\hline
\end{tabular}

Foram realizados testes estatísticos para as fibras de PDMS e Poliacrilato através da 
ferramenta Anova: fator único do software Excel. Constatou-se que as fibras são estatisticamente iguais mesmo após a realização de sucessivas análises, mostrando assim a confiabilidade da técnica de micro extração em fase solida (SPME) e a durabilidade das fibras.

\section{CONCLUSÃO}

Embora a dependência dos parâmetros de Flory-Huggins com a temperatura não seja clara, para esses polímeros, os valores de $\chi$ para os sistemas de etanol, tanto em PDMS quanto em PA, apresentaram-se inversamente proporcionais à temperatura, enquanto que o sistema de metanol-PDMS mostrou-se diretamente proporcional. Não foi possível comparar os resultados experimentais com os dados da literatura porque não foram encontrados valores do parâmetro de FloryHuggins para os sistemas aqui estudados, ou seja, estes valores são inéditos. A técnica de SPME apresentou-se, portanto, como uma técnica rápida e de execução simples, quando comparadas às técnicas de viscosidade intrínseca e de cromatografia inversa, para obtenção de parâmetros termodinâmicos para este tipo de mistura. Além disso, mostrou-se plenamente satisfatória.

\section{REFERÊNCIAS BIBLIOGRÁFICAS}

BRANDRUP, J.; IMMERGUT, E. H.; GRULKE, E. A. Polymer Handbook, $4^{\text {a }}$ edição. Nova York: Wiley, 1998.

CONDER, J. R.; YOUNG, C. L. Physicochemical measurements by gas chromatography. Journal of High Resolution Chromatography, v.3, p.486, 1979.

FLORY, P. J. Principles of Polymer Chemistry, $1^{\text {a }}$ edição. Ithaca: Cornell University Press, 1953.

FONSECA, D. B., COELHO, G. L. V. Determinação do coeficiente de atividade na diluição infinita $\left(\gamma^{\infty}\right)$ através da micro-extração em fase sólida (SPME). Química Nova, v.30, p. 1606-1608.

HAMMER , W. E.; DE LIGNY, C. L. Determination of Flory-Huggins $\chi$ parameter, the heat of dilution, and the excess heat capacity of some alkanes in polidimethylsiloxane by gas chromatography. Journal of Polymer Science, v.12, p.2065-2074, 1974.

HEBERGER, K.; MILCZEWSKA K.; VOELKEL, A. Principal component analysis of polymer-solvent and fillersolvent interactions by inverse gas chromatography. Colloids and Surfaces A: Physicochemical Engineering Aspects, v.260, p.29-37, 2005.

KALYUZHNYI, N. E.; YAMPOL'SKILL, $\mathrm{Yu}$. P. Chromatographic determination of the Flory-Huggins parameter and solubility parameter of polyvinyltrimethylsilane. Polymer Science U.S.S.R., v.31, p.1883-1888, 1989.

MARTOS, P. A.; SARAULLO, A; PAWLISZYN, J. Estimation of air/coating distribution coefficients for solid phase microextraction using retention indexes from linear temperature- programmed capillary gas chromatography: application to the sampling and analysis of total petroleum hydrocarbons in air. Analytical Chemistry, v.69, p.402408, 1997.

PAIK, J. S.; WRITER, M. S. Prediction of Flavor Sorption Using the FloryHuggins Equation. Journal of Agricultural and Food Chemistry, v.43, p.175-178, 1995.

VALENTE, A. L. P.; AUGUSTO, F. Microextração por fase sólida. Química Nova, v.23, p.523-530, 2000.

VOELKEL, A.; STRZEMIECKA, B.; ADAMSKA, K.; MILCZEWSKA, K. Inverse gas chromatography as a source of physiochemical data. Journal of Chromatography A, v.1216, p.15511566, 2009.

\section{AGRADECIMENTOS}

Agradecemos ao $\mathrm{CNPq}$ pela bolsa de Iniciação Científica e a FAPERJ pelo financiamento do projeto. 